\title{
Video-Based 3-Dimensional Surface Imaging
}

National Cancer Institute

\section{Source}

National Cancer Institute. Video-Based 3-Dimensional Surface Imaging. NCI Thesaurus.

Code C116501.

The use of recorded video images during stereotactic procedures to detect motioninduced misalignment, and allow for precise repositioning of the patient. 